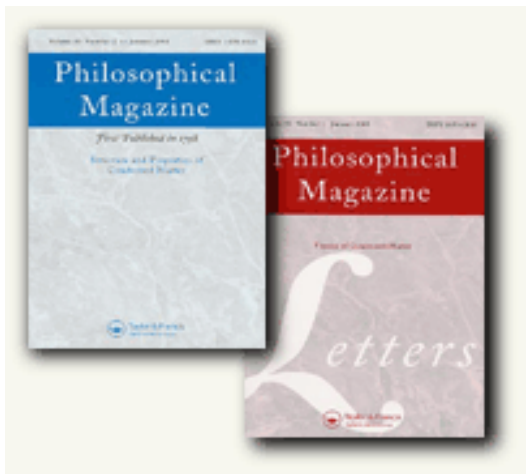

\title{
A three-dimensional dislocation field crystal plasticity approach applied to miniaturized structures
}

\begin{tabular}{|r|l|}
\hline Journal: & Philosophical Magazine \& Philosophical Magazine Letters \\
\hline Manuscript ID: & TPHM-06-May-0135.R1 \\
\hline Dournal Selection: & Philosophical Magazine \\
\hline Author: & 26-Jun-2006 \\
\hline Complete List of Authors: & $\begin{array}{l}\text { Bayley, Christopher; NIMR } \\
\text { Brekelmans, Marcel; Eindhoven University of Technology, } \\
\text { Mechanical Engineering } \\
\text { Geers, Marc; Eindhoven University of Technology, Mechanical } \\
\text { Engineering }\end{array}$ \\
\hline Keywords: & dislocation mechanics, grain boundary interfaces, micromechanics \\
\hline Keywords (user supplied): & crystal plasticity, miniaturization, strain gradient \\
\hline & \\
\hline $\begin{array}{l}\text { Note: The following files were submitted by the author for peer review, but cannot be converted } \\
\text { to PDF. You must view these files (e.g. movies) online. }\end{array}$ \\
\hline $\begin{array}{l}\text { PhysMag_spec_issue.tex } \\
\text { PhysMag_spec_issue.bib }\end{array}$ \\
\hline
\end{tabular}

\section{ScholaronE \\ Manuscript Central}




\title{
A three-dimensional dislocation field crystal plasticity approach applied to miniaturized structures
}

\author{
C.J. Bayley ${ }^{\star}$, W.A.M. Brekelmans $\dagger$, and M.G.D. Geers $\dagger$ \\ * Netherlands Institute for Metals Research \\ Mekelweg 2, 2628 CD Delft, The Netherlands \\ (now at Defence Research Development Canada) \\ ${ }^{\dagger}$ Eindhoven University of Technology, \\ Department of Mechanical Engineering, \\ P.O. Box 513, 5600 MB Eindhoven, the Netherlands
}

\begin{abstract}
Material size effects are predicted for idealized planar micromanufactured structures, as a consequence of the competitive contributions of strain gradient strengthening and loss of microstructural constraints with diminishing dimensions, assuming a constant grain size. Simulations are carried out using a 3D strain gradient crystal plasticity model which intrinsically accounts for the influence of differently oriented crystals within the material. By distinguishing between different crystallographic slip boundary conditions, the influences of surface layer passivity, internal grain boundaries and back stresses are assessed under externally applied in-plane tension and through-thickness bending loading conditions. Analyses are carried out on samples with a size that is representative of micromanufacturing processes. The simulations reveal a competitive process between first-order constraints, generally inducing a weakening behaviour as the number of grains decreases, and secondorder strengthening resulting from the strain gradients.
\end{abstract}

Key words: strain gradient, crystal plasticity, back stress, grain boundaries, dislocation fields, geometrically necessary dislocations, miniaturization, MEMS, micromanufacturing

\section{Introduction}

With ever successive electronic device miniaturization, component dimensions are approaching those of the underlying microstructure. This is particularly evident in the field of Miniaturized Electro Mechanical Systems (MEMS), which are used in applications such as tunable capacitors and pumps in micro-fluidic applications such as lab-on-a-chip. Recently, the World Technology Evaluation Center published a report on research and development in micromanufacturing [1], which covers length scales that are somewhat larger than those of most MEMS applications 
but are not based on lithographic-based processes. Even within this range of component length scales $(\sim 100 \mu \mathrm{m})$, microstructural engineering becomes increasingly important, as the global behaviour of the metallic components may be dominated by the response of individual grains. Clearly, this behaviour cannot be described by a macroscopic (averaging) continuum approach that ignores individually oriented grains and their boundaries. Therefore, understanding the microstructural phenomena is crucial in assessing the mechanical response of these components.

The component size and microstructural features of a typical miniaturized test sample are visible in Figure 1, which shows an FCC beam-type specimen used to experimentally quantify the microstructural characteristics of processed micromanufactured systems. The miniaturized component in Figure 1a, is manufactured with

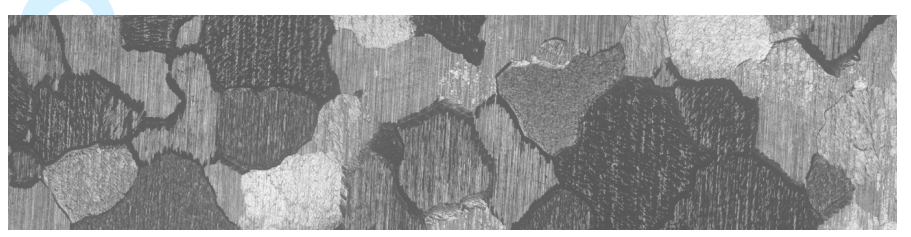

(a)

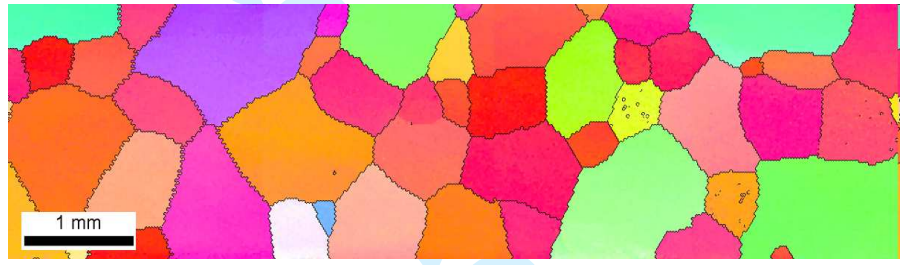

(b)

Fig. 1. a) Micromanufactured sample with large through-thickness grains and a thickness of $300 \mu \mathrm{m} . \mathrm{b})$ Typical orientation image map, revealing the relative crystallographic mismatches between adjacent grains along the planar surface of one of the samples.

a thickness of $300 \mu \mathrm{m}$, with only a single through-thickness grain. The outlines of some of the grains are clearly visible, reflecting a typical ratio between specimen size and grain size, which is of interest in the present work. The different orientations between the constituent grains are qualitatively shown in Figure 1b. Depending on the processing conditions, micromanufactured components may have an irregular crystallography with a near random or pronounced texture. The implication of the crystallographic scatter within the sample may induce a large variance in global mechanical properties, and hence potentially unacceptable rejection rates of individual components which fall outside the allowable tolerances. Whereas a lot of work has been done at the submicron length scale, examining for instance the formation of boundary layers, less is known about what impact the grain size and number of grains have on the mechanical behaviour of micromanufactured components. In this circumstance the ratio between the grain size and the component size is a governing parameter.

In order to accurately predict the mechanical response of such miniaturized com- 
ponents, the material model must, as opposed to a continuum plasticity model, account for the influence that individual grains have on the aggregate behaviour. The fundamental assumption that the material behaves as a homogeneous continuum is invalid as dimensions diminish and the statistical phenomenon that individual grains dominate the component behaviour becomes increasingly important. An appropriate point of departure for an analysis is a constitutive rate-dependent crystal plasticity theory $[2,3]$ where the response of each individual crystal is included in the definition. However, not only must the response of each crystal be included, but also a description of what happens at their boundaries. Internal and external boundaries form obstacles to crystallographic slip which must be overcome with an increased applied load. Within the current material model, the obstructions at internal boundaries are taken into account by inhibiting crystallographic slip across the boundary through the accumulation of geometrically necessary dislocations (GNDs), which are required to accommodate plastic slip gradients [4]. The GNDs contribute to the slip system resistance, while back stresses associated with the GND stress fields, provide kinematic hardening. The influence of the GNDs is particularly apparent in cases which develop macroscopic strain gradients such as shearing, torsion and bending $[5,6,7,8]$. However, by neglecting the development of GNDs completely, this strain-gradient crystal plasticity model reverts back to a first-order crystal plasticity model, making this model particularly well suited to examine the competitive relationship between first order weakening and second order strengthening when the number of grains decreases.

\begin{abstract}
Experimentally, material size effects have been examined in both micro-beam bending of bars and micro-torsion of wires $[9,10]$. These observations of size dependence have also been predicted numerically $[5,6,7,11,12,13,14,15,16,17,18]$. However, the investigations typically involve scaling of both the geometry and the microstructure simultaneously, confounding the influence of the size effect. Strictly speaking, only the external component geometry should be scaled during miniaturization while maintaining a constant internal microstructure, as physically relevant for the specimen size effect. Empirical and numerical evidences suggest that smaller is stronger, however, this neglects contributions due to the weakening influence of reduced crystallographic constraints as the number of grains within the component are reduced as in the case of micromanufactured components. This subject was advocated experimentally in [19, 20, 21,22], and justifies an analysis in the considered range of length scales. Using a 3D second-order strain gradient crystal plasticity approach, this paper examines the qualitative relevance and competitive influence between gradient strengthening and weakening, under imposed uniaxial tension and bending with 'large' grains and specimen dimensions in the range of $100 \mu \mathrm{m}$ to $1 \mathrm{~mm}$.
\end{abstract}




\section{Strain gradient crystal plasticity material model}

The adopted second-order strain gradient crystal plasticity model is next briefly outlined, which was initially developed by Evers et al. [6,11]. A key feature of the model is the incorporation of both geometrically necessary and statistically stored dislocation densities which are used to affect the crystallographic slip resistance, while the gradients of the GND field are used to determine the internal stress state that acts as a dislocation induced back stress.

In order to consistently represent the different scalar, vector and tensorial quantities, the following notation convention is pursued: scalar quantities are written in italic symbols i.e. $b$, vectorial quantities are written in bold italics i.e. $s_{0}^{\alpha}$ and $n_{0}^{\alpha}$, and matrices and second tensors are written in an upright sans-serif font i.e. $F_{e}$ and $S$, while fourth order tensors are expressed as $\mathbb{C}$. Tensorial notation is used throughout whereby $\cdot$ represents an inner product and : represents the double inner product.

As a classical point of departure, the deformation gradient tensor $F$, is multiplicatively decomposed into its elastic part $F_{e}$ and a plastic part $F_{p}$, which is visualized in Figure 2, according to:

$$
\mathrm{F}=\mathrm{F}_{\mathrm{e}} \cdot \mathrm{F}_{\mathrm{p}}
$$

The plastic contribution $F_{p}$ refers to the deformation from the initial reference configuration to the intermediate stress-free configuration. This stress-free configuration can be considered to develop from the reference configuration solely by plastic shearing along the active slip planes of the crystal lattice through crystallographic slip, leaving the orientations of the slip systems unaltered. The elastic part of the deformation tensor rotates and stretches the plastically deformed material into the current configuration.

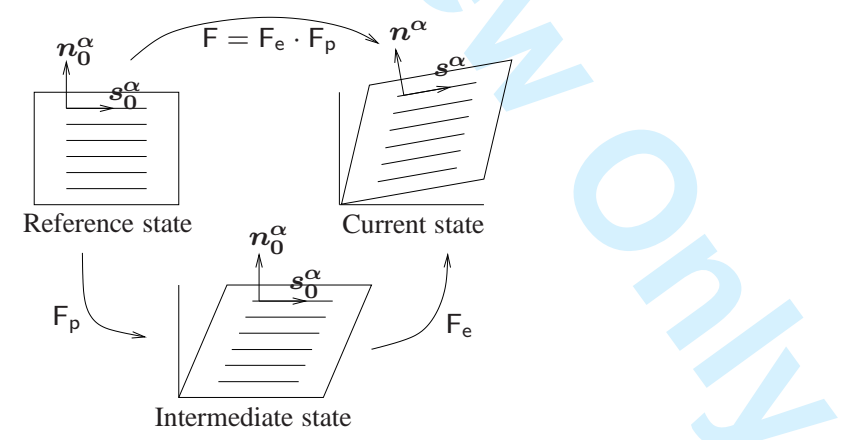

Fig. 2. Multiplicative decomposition of the deformation into a plastic and elastic part.

In Figure 2, an arbitrary slip system is labelled by a superscript $\alpha$, with $\alpha=$ $1,2 \ldots, n_{s}$ where $n_{s}$ is the total number of slip systems. The individual slip systems are defined according to Table 1 for an FCC crystal. In the reference state, a slip system $\alpha$ is identified by unit vectors representing the slip plane normal $\boldsymbol{n}_{0}^{\alpha}$ and the slip direction $s_{0}^{\alpha}$. 
Table 1

Slip system $(\alpha)$ and dislocation $(\xi)$ indices with their corresponding normal $\left(\boldsymbol{n}_{\mathbf{0}}^{\boldsymbol{\alpha}}\right)$ and slip $\left(s_{0}^{\boldsymbol{\alpha}}\right)$ direction unit vectors for an FCC crystal.

\begin{tabular}{ccccc}
$\xi$ & $\alpha$ & Dislocation type & $s_{0}^{\alpha}$ & $n_{0}^{\alpha}$ \\
\hline 1 & 1 & edge & $\frac{1}{\sqrt{2}}[\overline{1} 10]$ & $\frac{1}{\sqrt{3}}(111)$ \\
2 & 2 & edge & $\frac{1}{\sqrt{2}}[10 \overline{1}]$ & $\frac{1}{\sqrt{3}}(111)$ \\
3 & 3 & edge & $\frac{1}{\sqrt{2}}[0 \overline{1} 1]$ & $\frac{1}{\sqrt{3}}(111)$ \\
4 & 4 & edge & $\frac{1}{\sqrt{2}}[\overline{1} \overline{1} 0]$ & $\frac{1}{\sqrt{3}}(1 \overline{1} \overline{1})$ \\
5 & 5 & edge & $\frac{1}{\sqrt{2}}[101]$ & $\frac{1}{\sqrt{3}}(1 \overline{1} \overline{1})$ \\
6 & 6 & edge & $\frac{1}{\sqrt{2}}[01 \overline{1}]$ & $\frac{1}{\sqrt{3}}(1 \overline{1} \overline{1})$ \\
7 & 7 & edge & $\frac{1}{\sqrt{2}}[110]$ & $\frac{1}{\sqrt{3}}(\overline{1} 1 \overline{1})$ \\
8 & 8 & edge & $\frac{1}{\sqrt{2}}[\overline{1} 01]$ & $\frac{1}{\sqrt{3}}(\overline{1} 1 \overline{1})$ \\
9 & 9 & edge & $\frac{1}{\sqrt{2}}[0 \overline{1} \overline{1}]$ & $\frac{1}{\sqrt{3}}(\overline{1} 1 \overline{1})$ \\
10 & 10 & edge & $\frac{1}{\sqrt{2}}[1 \overline{1} 0]$ & $\frac{1}{\sqrt{3}}(\overline{1} \overline{1} 1)$ \\
11 & 11 & edge & $\frac{1}{\sqrt{2}}[\overline{1} 0 \overline{1}]$ & $\frac{1}{\sqrt{3}}(\overline{1} \overline{1} 1)$ \\
12 & 12 & edge & $\frac{1}{\sqrt{2}}[011]$ & $\frac{1}{\sqrt{3}}(\overline{1} \overline{1} 1)$ \\
13 & 4 or 7 & screw & $\frac{1}{\sqrt{2}}[110]$ & $\frac{1}{\sqrt{3}}(1 \overline{1} \overline{1})$ or $\frac{1}{\sqrt{3}}(\overline{1} 1 \overline{1})$ \\
14 & 5 or 11 & screw & $\frac{1}{\sqrt{2}}[101]$ & $\frac{1}{\sqrt{3}}(1 \overline{1} \overline{1})$ or $\frac{1}{\sqrt{3}}(\overline{1} \overline{1} 1)$ \\
15 & 9 or 12 & screw & $\frac{1}{\sqrt{2}}[011]$ & $\frac{1}{\sqrt{3}}(\overline{1} 1 \overline{1})$ or $\frac{1}{\sqrt{3}}(\overline{1} \overline{1} 1)$ \\
16 & 1 or 10 & screw & $\frac{1}{\sqrt{2}}[\overline{1} 10]$ & $\frac{1}{\sqrt{3}}(111)$ or $\frac{1}{\sqrt{3}}(\overline{1} \overline{1} 1)$ \\
17 & 2 or 8 & screw & $\frac{1}{\sqrt{2}}[10 \overline{1}]$ & $\frac{1}{\sqrt{3}}(111)$ or $\frac{1}{\sqrt{3}}(\overline{1} 1 \overline{1})$ \\
18 & 3 or 6 & screw & $\frac{1}{\sqrt{2}}[0 \overline{1} 1]$ & $\frac{1}{\sqrt{3}}(111)$ or $\frac{1}{\sqrt{3}}(1 \overline{1} \overline{1})$
\end{tabular}

The elastic behaviour is considered with respect to the fictitiously unloaded configuration defined by the plastic deformation gradient tensor $F_{p}$. A hyper-elastic formulation is selected where the second Piola-Kirchhoff stress tensor $\mathrm{S}$ is expressed in the (elastic) Green-Lagrange strain tensor $E_{e}$ defined with respect to the intermediate configuration according to:

$$
\mathrm{S}=\mathbb{C}: \mathrm{E}_{\mathrm{e}} \quad \text { with } \quad \mathrm{E}_{\mathrm{e}}=\frac{1}{2}\left(\mathrm{~F}_{\mathrm{e}}^{T} \cdot \mathrm{F}_{\mathrm{e}}-\mathrm{I}\right)
$$

with I the second order identity tensor, while the stress tensor $S$ is defined by:

$$
\mathrm{S}=\mathrm{F}_{\mathrm{e}}^{-1} \cdot \tau \cdot \mathrm{F}^{-T} \quad \text { with } \quad \tau=J_{e} \sigma
$$

where $\tau$ is the Kirchhoff stress tensor, $\sigma$ the Cauchy stress tensor and $J_{e}=\operatorname{det}\left(\mathrm{F}_{\mathrm{e}}\right)=$ $\operatorname{det}(\mathrm{F})$ the volume change ratio while for $\mathbb{C}$ the fourth order isotropic elasticity tensor is taken.

For a given slip system $\alpha$ the resolved shear stress $\tau^{\alpha}$ (also called the Schmid stress) in the intermediate state can be determined through:

$$
\tau^{\alpha}=\boldsymbol{s}_{0}^{\alpha} \cdot \mathrm{S} \cdot \boldsymbol{n}_{0}^{\alpha}
$$


The plastic velocity gradient tensor in the intermediate configuration $L_{p}$ is composed of the contributions over the slip systems:

$$
\mathrm{L}_{p}=\sum_{\alpha=1}^{n_{s}} \dot{\gamma}^{\alpha} \boldsymbol{s}_{0}^{\alpha} \boldsymbol{n}_{0}^{\alpha}
$$

where the summation runs over the total number $n_{s}$ of slip systems and where $\dot{\gamma}^{\alpha}$ is the yet to be determined slip rate quantity on each individual slip system $\alpha$. The plastic velocity gradient tensor is related to the plastic deformation gradient tensor according to:

$$
\dot{\mathrm{F}}_{\mathrm{p}}=\mathrm{L}_{\mathrm{p}} \cdot \mathrm{F}_{\mathrm{p}}
$$

The connection between the single crystal kinematics and the underlying dislocation density development is accomplished through a visco-plastic power-law which relates the slip rates to the effective shear stress $\tau_{\text {eff }}^{\alpha}$ and the slip system resistance $s^{\alpha}$ according to:

$$
\dot{\gamma}^{\alpha}=\dot{\gamma}_{0}\left(\frac{\left|\tau_{e f f}^{\alpha}\right|}{s^{\alpha}}\right)^{1 / m} \exp \left[-\frac{G_{0}}{k T}\left(1-\frac{\left|\tau_{\text {eff }}^{\alpha}\right|}{s^{\alpha}}\right)\right] \operatorname{sign}\left(\tau_{\text {eff }}^{\alpha}\right)
$$

with $\dot{\gamma}_{0}$ and $m$ material parameters, representing the reference plastic shear rate and the rate sensitivity, respectively. $T$ and $k$ are the absolute temperature and Boltzmann's constant, respectively, and $G_{0}$ is the thermal activation energy necessary to activate dislocation motion. With a small value of $m$ (i.e. $m=0.1) \dot{\gamma}^{\alpha}$ remains negligible unless $\left|\tau_{\text {eff }}^{\alpha}\right|$ is close to $s^{\alpha}$ or larger (reflecting the 'viscous' activation of the slip system).

The effective shear stress $\tau_{\text {eff }}^{\alpha}$ constitutes the driving force for crystallographic slip through dislocation motion on slip system $\alpha$ and is determined as the difference between the externally imposed resolved shear stress $\tau^{\alpha}$, see equation (4), and the yet to be defined resolved back stress $\tau_{b}^{\alpha}$ according to:

$$
\tau_{e f f}^{\alpha}=\tau^{\alpha}-\tau_{b}^{\alpha}
$$

The slip system resistance $\left(s^{\alpha}\right)$ is a measure of the impedance of dislocation motion on the slip systems by the formation of short-range interactions between all dislocations. Physically, dislocations are discrete loops but are represented here by a continuous field of dislocations with either an edge or screw nature. In contrast to more phenomenological crystal plasticity models which relate the slip resistance to the history of the plastic shear on all slip systems, here the slip resistance $s^{\alpha}$ on slip system $\alpha$ is expressed as a function of both the dislocation densities $\rho_{G N D}^{\xi}$ and $\rho_{S S D}^{\xi}$, with the superscript $\xi$ denoting the dislocation type as labelled in Table 1 . The slip system resistance includes the contribution of both the SSDs and GNDs 
according to:

$$
s^{\alpha}=G b \sqrt{\sum_{\xi=1}^{12} \mathrm{~A} \alpha \xi\left|\rho_{S S D}^{\xi}\right|+\sum_{\xi=1}^{18} \mathrm{~A} \alpha \xi\left|\rho_{G N D}^{\xi}\right|}
$$

where $G$ is the shear modulus, $b$ the magnitude of the Burgers vector, and $\mathrm{A}^{\alpha \xi}$ is a component of an interaction matrix which represents the strength of the interactions between slip systems as determined by Franciosi and Zaoui [23]. The six relevant interaction coefficients corresponding to self hardening, coplanar, Hirth lock, glissile junction, Lomer Cottrell lock, and cross slip are further defined by Arsenlis and Parks [24]. Note that equation (9) incorporates edge SSDs and edge GNDs since they are the major source of hardening in FCC, whereas screw GNDs are accounted for as well since at least one screw population is needed to accommodate certain strain gradients with geometrically necessary dislocations.

\section{Internal stress formulations}

Because dislocations disturb the regularity of crystal lattices they constitute a source of internal stress. For statistically stored dislocations, which usually have a random orientation, the net internal stress contribution will be negligibly small. However, geometrically necessary dislocations may cause a significant internal stress state, which can be estimated from the self-equilibrating elastic stress fields associated with individual edge and screw dislocations [25]. The evaluation of the internal stress was carried out by Evers et al. $[6,11]$ for the dislocation fields distributed on each of the slip systems, thereby ignoring the latent contributions originating from other slip systems. This is presently improved by considering all stress components of all GND fields on all slip systems [5]. Through analytical integration over a cylindrical domain with a finite radius $R$, within which dislocations are considered to contribute to the back stress, the following internal stress state can be derived for a field of edge dislocations:

$$
\begin{aligned}
\sigma_{\mathrm{e}}^{\mathrm{int}}=\frac{G b R^{2}}{8(1-\nu)} \sum_{\xi=1}^{12} \boldsymbol{\nabla}_{\boldsymbol{0}} \rho_{G N D}^{\xi} \cdot[ & 3 \boldsymbol{n}_{0}^{\xi} \boldsymbol{s}_{0}^{\xi} \boldsymbol{s}_{0}^{\xi}+\boldsymbol{n}_{0}^{\xi} \boldsymbol{n}_{0}^{\xi} \boldsymbol{n}_{0}^{\xi}+4 \nu \boldsymbol{n}_{0}^{\xi} \boldsymbol{p}_{0}^{\xi} \boldsymbol{p}_{0}^{\xi} \\
& \left.-\boldsymbol{s}_{0}^{\xi} \boldsymbol{s}_{0}^{\xi} \boldsymbol{n}_{0}^{\xi}-\boldsymbol{s}_{0}^{\xi} \boldsymbol{n}_{0}^{\xi} \boldsymbol{s}_{0}^{\xi}\right]
\end{aligned}
$$

while for a field of screw dislocations, the resulting internal stress can be written as:

$$
\sigma_{\mathrm{s}}^{\mathrm{int}}=\frac{G b R^{2}}{4} \sum_{\xi=13}^{18} \boldsymbol{\nabla}_{\mathbf{0}} \rho_{G N D}^{\xi} \cdot\left[-\boldsymbol{n}_{0}^{\xi} \boldsymbol{s}_{0}^{\xi} \boldsymbol{p}_{0}^{\xi}-\boldsymbol{n}_{0}^{\xi} \boldsymbol{p}_{0}^{\xi} \boldsymbol{s}_{0}^{\xi}+\boldsymbol{p}_{0}^{\xi} \boldsymbol{s}_{0}^{\xi} \boldsymbol{n}_{0}^{\xi}+\boldsymbol{p}_{0}^{\xi} \boldsymbol{n}_{0}^{\xi} \boldsymbol{s}_{0}^{\xi}\right]
$$

where $\boldsymbol{n}_{0}^{\xi}$ and $\boldsymbol{s}_{0}^{\xi}$ define the slip system normal and slip direction, $\boldsymbol{p}_{0}^{\xi}=\boldsymbol{s}_{0}^{\xi} \times \boldsymbol{n}_{0}^{\xi}$ associated with the $\xi$ dislocation listed in Table 1 , and $\nabla_{0}$ is the gradient of the 
dislocation density related to the reference configuration. For the screw dislocations, either one of the two slip systems defined in Table 1 results in an equivalent internal stress, and therefore the choice of the slip system associated with a screw dislocation is irrelevant. A detailed assessment of different types of internal stress formulations is given in [5].

Analogous to resolving the Schmid stress on each slip system defined in equation (4), the back stress on each slip system is resolved from the dislocation induced internal stress tensor according to:

$$
\tau_{b}^{\alpha}=-\boldsymbol{s}_{0}^{\alpha} \cdot\left(\sigma_{\mathrm{s}}^{\mathrm{int}}+\sigma_{\mathrm{e}}^{\mathrm{int}}\right) \cdot \boldsymbol{n}_{0}^{\alpha}
$$

where the minus sign in the right-hand side of equation (12) is introduced to provide consistency with the definition of the back stress as introduced in equation (8). Latent hardening, herein defined as the hardening on a secondary slip system caused by dislocation gradients on a primary slip system, is incorporated by summation over $\xi=1,2,3, \ldots, 12$ for the edge dislocations and $\xi=13,14, \ldots, 18$ for the screw dislocations, respectively.

\section{Dislocation density evolution}

The evolutions of the 12 edge SSD densities of an FCC material, which are required for equation (9), are based on the balance between accumulation and annihilation rates according to the references $[6,24,26]$ and [11]:

$$
\dot{\rho}_{S S D}^{\alpha}=\frac{1}{b}\left(\frac{1}{L^{\alpha}}-2 y_{c} \rho_{S S D}^{\alpha}\right)\left|\dot{\gamma}^{\alpha}\right| \quad \text { with: } \quad \rho_{S S D}^{\alpha}(t=0)=\rho_{S S D_{0}}^{\alpha}
$$

The accumulation rate (first term in the right-hand side of equation (13)) is governed by the average dislocation segment length of mobile dislocations (SSDs) on system $\alpha$, denoted by $L^{\alpha}$, which is directly related to the current dislocation state according to:

$$
L^{\alpha}=\frac{K}{\sqrt{\sum_{\xi=1}^{12} \mathrm{H}^{\alpha \xi}\left|\rho_{S S D}^{\xi}\right|+\sum_{\xi=1}^{18} \mathrm{H}^{\alpha \xi}\left|\rho_{G N D}^{\xi}\right|}}
$$

In this expression the dimensionless coefficients $\mathrm{H}^{\alpha \xi}$, represent the mutual immobilization between dislocations of different slip systems, structured analogously to the coefficients $A^{\alpha \xi}$ introduced in equation (9), yet with different values. Furthermore, the annihilation rate (second term in the right-hand side of equation (13)) is controlled by the critical annihilation length $y_{c}$, a material parameter characterizing the average distance between dislocations of opposite signs which triggers spontaneous neutralization. Note that equation (13) does not imply that GNDs are not mobile. Individual dislocations cannot discriminate between being SSD or GND. 
In fact, the GND fraction of the total dislocation population is determined geometrically. Nevertheless, GNDs do contribute to the production of SSDs, as clearly expressed by equation (14).

Gradients in the plastic deformation within crystalline materials give rise to socalled geometrically necessary dislocations in order to restore lattice continuity in the crystals. With the knowledge of the crystalline orientation in relation to the plastic deformation gradient, the type of dislocation needed to preserve this continuity can be determined.

Considering one of the slip systems, only slip gradients in the plane of the slip system give rise to an incompatibility that will lead to GNDs. Slip gradients in the direction of the slip will be accommodated by edge dislocations while gradients in the slip plane perpendicular to the slip direction induce screw dislocations. The relationship between the gradient (with respect to the undeformed reference configuration) of the plastic slip $\gamma^{\alpha}$ on a slip system $\alpha$ (with $\alpha=\xi=1,2, \ldots, 12$ for FCC material) and the associated edge GND density takes the following form, see Ashby [4]:

$$
\rho_{G N D}^{\xi}=\rho_{G N D_{0}}^{\xi}-\frac{1}{b} \nabla_{0} \gamma^{\xi} \cdot \boldsymbol{s}_{0}^{\xi}
$$

where an initial value of the GND density has been introduced to account for initially present geometrical lattice distortions (i.e. at small angle grain boundaries, see Evers et al. [11]). The screw GND densities for $\xi=13,14, \ldots, 18$, due to slip gradients can effectively be written as:

$$
\rho_{G N D}^{\xi}=\rho_{G N D_{0}}^{\xi}+\frac{1}{b}\left(\nabla_{0} \gamma^{\alpha_{1}} \cdot \boldsymbol{p}_{0}^{\alpha_{1}}+\nabla_{0} \gamma^{\alpha_{2}} \cdot \boldsymbol{p}_{0}^{\alpha_{2}}\right)
$$

with $\alpha_{1}$ and $\alpha_{2}$ indicating the two slip systems associated with each screw GND, as listed in Table 1.

\section{Finite element implementation}

In order to systematically compute an approximate solution of the entire set of strongly non-linear and coupled equations for an arbitrary geometry and boundary conditions, the previously described strain gradient crystal plasticity framework is implemented within the finite element method at the integration point level. A mixed formulation is thereby used, in which both displacement fields and GND fields are discretized. Each integration point describes the enriched constitutive response of the lattice within the integration point volume, whereby individual grains consist of a number of finite elements.

As apparent from equations (15) and (16) the spatial variation of the crystallographic slip throughout the domain sets the GND densities, moreover, the evolution of the crystallographic slip rates (equation (7)) requires knowledge of the 
GND densities through the crystallographic resistance (equation (9)) and the internal stress (equations (10) and (11)). GND densities are therefore incorporated as a field variable, from which all relevant quantities can be determined along with the solution for the displacement field. Therefore, the 18 GND densities are treated as nodal unknowns in a similar manner as the 3 displacements, increasing the number of nodal degrees of freedom to 21. Essentially, the stress equilibrium equations, here formulated in terms of the first Piola-Kirchhoff stress tensor (nominal stress) denoted by P, and the GND density equations (15) and (16) constitute the basis to determine the unknown degrees of freedom.

The incremental calculation of the material model at the integration point level involves a number of steps which are outlined below. Input to the material model from the global FE solution are the estimates of the deformation gradient tensor $\mathrm{F}^{*}$ and the GND densities, both to be determined from the current estimates of the nodal degrees of freedom and interpolation at the integration points through the element shape functions. Not only does the material model return the associated integration point values of $\mathrm{P}^{*}$ and $\gamma^{* \alpha}$, but also the tangent moduli. Further details of the FEM solution can be found in the references [5, 11], where only 2D solutions were considered.

- Starting from an estimate for the incremental slip rates $\dot{\gamma}^{* \alpha}$, determine the plastic part of the deformation gradient tensor $F_{p}^{*}$ by integrating equation (6) to yield $\mathrm{F}_{\mathrm{p}}^{*}=\left(\mathrm{I}+\Delta t \mathrm{~L}_{\mathrm{p}}\right) \cdot \mathrm{F}_{\mathrm{p}_{t}}$ where $\mathrm{F}_{\mathrm{p}_{t}}$ denotes the deformation gradient tensor at the end of the previous (converged) increment and $\Delta t$ is the current time step.

- With $\mathrm{F}_{\mathrm{p}}^{*}$, and the current deformation gradient tensor $\mathrm{F}^{*}$, determine the associated elastic deformation gradient tensor $F_{e}^{*}$ with equation (1). Subsequently compute the second Piola-Kirchhoff stress tensor $S^{*}$ using equation (2). Use this result to determine the resolved shear stresses $\tau^{* \alpha}$ on the slip systems $\alpha$ by applying equation (4).

- Determine the first Piola-Kirchhoff stress tensor P* according to:

$$
\mathrm{P}^{*}=\tau^{*} \cdot \mathrm{F}^{*(-T)}=\mathrm{F}_{\mathrm{e}}^{*} \cdot \mathrm{S}^{*} \cdot \mathrm{F}_{\mathrm{e}}^{*} \cdot \mathrm{F}^{*(-T)}=\mathrm{F}_{\mathrm{e}}^{*} \cdot \mathrm{S}^{*} \cdot \mathrm{F}_{\mathrm{p}}^{*(-T)}
$$

where it is recalled that the second Piola-Kirchhoff stress tensor $\mathrm{S}^{*}$ was related to the intermediate configuration while the first Piola-Kirchhoff stress tensor $\mathrm{P}^{*}$ is defined with respect to the undeformed reference state.

- Determine the dislocation induced internal stress tensors from the GND density fields defined by the nodal values $\rho_{G N D}^{* \xi}$ using the equations (11) and (10) and then calculate the back stresses $\tau_{b}^{* \alpha}$ on the slip systems with equation (12). From $\tau^{* \alpha}$ and $\tau_{b}^{* \alpha}$ determine the effective shear stresses with equation (8).

- Estimate $\rho_{S S D}^{* \xi}$ by integrating equation (13), and together with $\rho_{G N D}^{* \xi}$, the slip system resistances, $s^{* \alpha}$, can be calculated from equation (9).

- Evaluate the right-hand side of the slip law equation (7). The results will deviate from the previous slip rate estimates $\dot{\gamma}^{* \alpha}$, the differences of which are used to correct the current iterative state. As long as the convergence norm is not yet 
reached, a next iteration step (restarting the procedure from the beginning) is executed.

\section{3D dislocation field analyses for micromanufactured samples}

Finite element models examining the competitive influence between strain gradient strengthening and mutual grain constraints are considered under conditions of in-plane tension and out-of-plane pure bending. These models aid in understanding size effects in metallic material where the crystallographic texture along with surface and grain boundaries influence the macroscopic behaviour, as observed experimentally.

To examine the constraining influence of differently oriented neighbouring grains qualitatively, FEM models were constructed to represent flat beam-like miniaturized components with varying width and thickness, but composed of identically shaped brick-like crystals. The material parameters for the models are representative of copper and adopted from the references $[5,11]$. The grains were arranged to form a rectangular volume extending 10 crystals in length with variable widths and thicknesses as shown in Figure 3. The configuration with only a single row of crystals (Figure 3a) models the performance of a miniaturized strut-like component, while the configuration with seven rows of crystals (Figure 3e) approaches a macroscopic plate-like geometry. Note that all samples have only a single grain across the thickness, consistent with the processed specimens shown in Figure 1. Since each crystal has a unique randomly selected crystallographic orientation, the grain boundaries between adjacent grains impose deformation constraints by obstructing plastic slip due to the mismatch of the mutual slip systems. Hence, the model with a single row of crystals (Figure 3a) has the least amount of constraint while a successively greater number of crystals in the width direction increases the constraint caused by neighbouring grains. The rectangular microstructures have dimensions of $100 \mu \mathrm{m}$ in both the length and width directions, but with thicknesses ranging from 75 to $300 \mu \mathrm{m}$. The response from one of these microstructures is compared to the response from a configuration with randomly shaped grains in order to assess the influence of the use of regularly shaped grains within the structure. The randomly shaped model (Figure 3f) has 49 individual grains and dimensions similar to the $500 \mu \mathrm{m}$ model (Figure 3d) with five cubic grains in the width direction. The cubic shaped grains are represented by 27 brick elements as shown in Figure 3 with either linear, selectively reduced integration brick elements (hex8) or quadratic brick elements (hex27), depending on the applied loading conditions. Linear brick elements (with selectively reduced integration to prevent locking) are used for the tensile models while quadratic brick elements are used to accurately capture the through-thickness bending, but uses a limited number of elements. A previous mesh sensitivity study [27] revealed that a limited influence of the mesh density for similarly sized elements under both bending and tension loading condi- 
tions. This relative mesh insensitivity is not surprising given that the model equations share a common structure with some continuum gradient plasticity models which have also been shown to be largely mesh-independent as well.

Displacement boundary conditions are specified for the nodes lying at both ends of the major axes, adequately prescribing the external loading conditions, including suppression of rigid body motion without additional (transverse) constraints. The tensile models utilize a constant thickness of $100 \mu \mathrm{m}$ but with variable widths, while the out-of-plane bending models utilize the specimen with a constant width of $300 \mu \mathrm{m}$ (represented by the configuration as shown in Figure 3c) but with variable thicknesses.

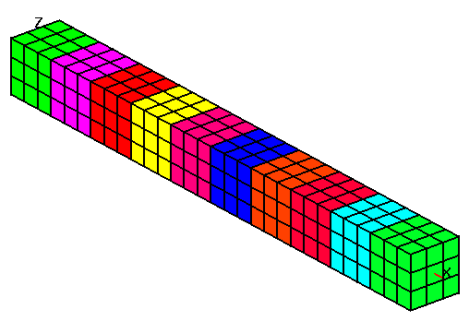

(a)

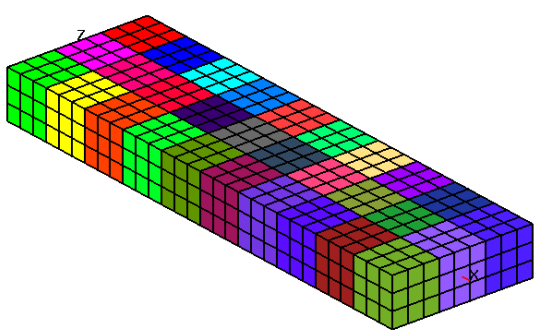

(c)

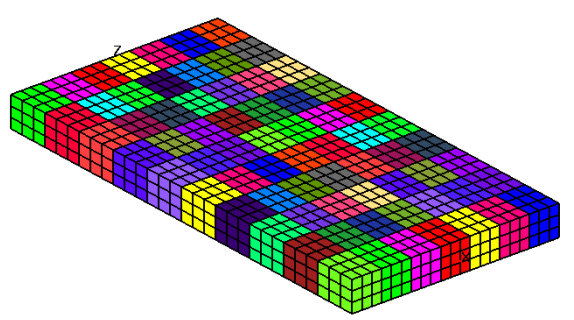

(e)

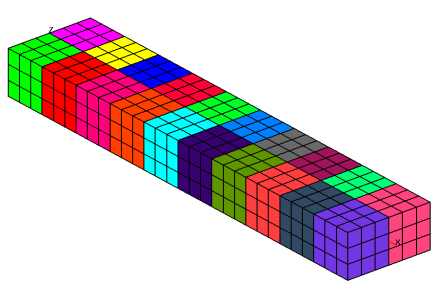

(b)

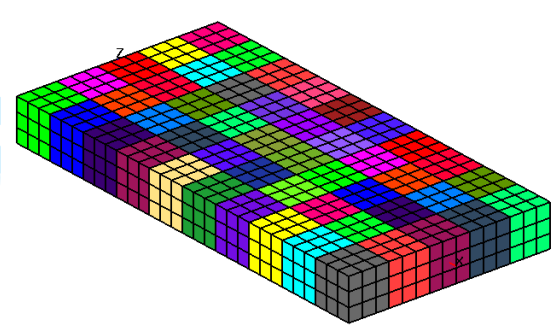

(d)

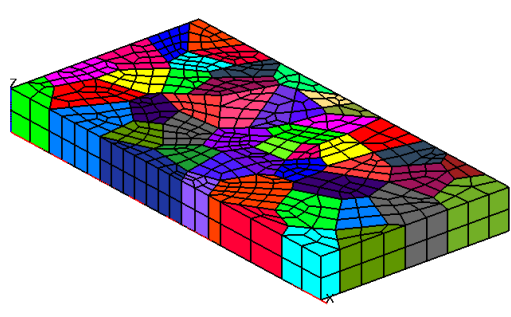

(f)

Fig. 3. a-e) Regular planar MEMS models with only single through thickness grains and variable widths used in the tensile simulations while f) presents a more realistic grain structure. The bending simulations are based on the mesh shown in c), but with variations in the thickness dimension.

To properly incorporate grain boundaries, grains are modelled as separate volumetric domains. Along the coinciding grain boundaries, double nodes are introduced to represent the mutual grain boundary. The displacement degrees of freedom of these double nodes are always mutually tied to preserve kinematic compatibility, 
while the GND degrees of freedom are uncoupled to allow for different dislocation densities at either side of the boundary. The plastic slip between grains is assumed to be fully obstructed which forces GNDs to accumulate at both adjacent grain boundaries. Where grain boundaries coincide with the specimen outer surface two different conditions are represented, suppressed plastic slip (modelling a hard oxide layer on the specimen surface) or free plastic slip (inducing a vanishing GND density at the specimen surface). By either inhibiting or permitting crystallographic slip in the specimen surface normal direction, upper and lower (stiffness) bounds of the specimen response are obtained. Additionally, to realize an absolute lower bound for reference purposes, calculations are also performed with a standard firstorder crystal plasticity approximation without any gradient effects (in which all GND effects are ignored).

For each model geometry, a random selection of grain orientations, sampled from a random texture was taken, which was repeated at least five times in order to quantitatively assess the influence of grain statistics on the macroscopic behaviour. Emphasis in the analysis is on (1) the 3D effects; (2) the influence of competing size effects at the considered (coarse) scale; (3) a qualitative assessment of the constraints at internal and external boundaries.

\subsection{The tensile case}

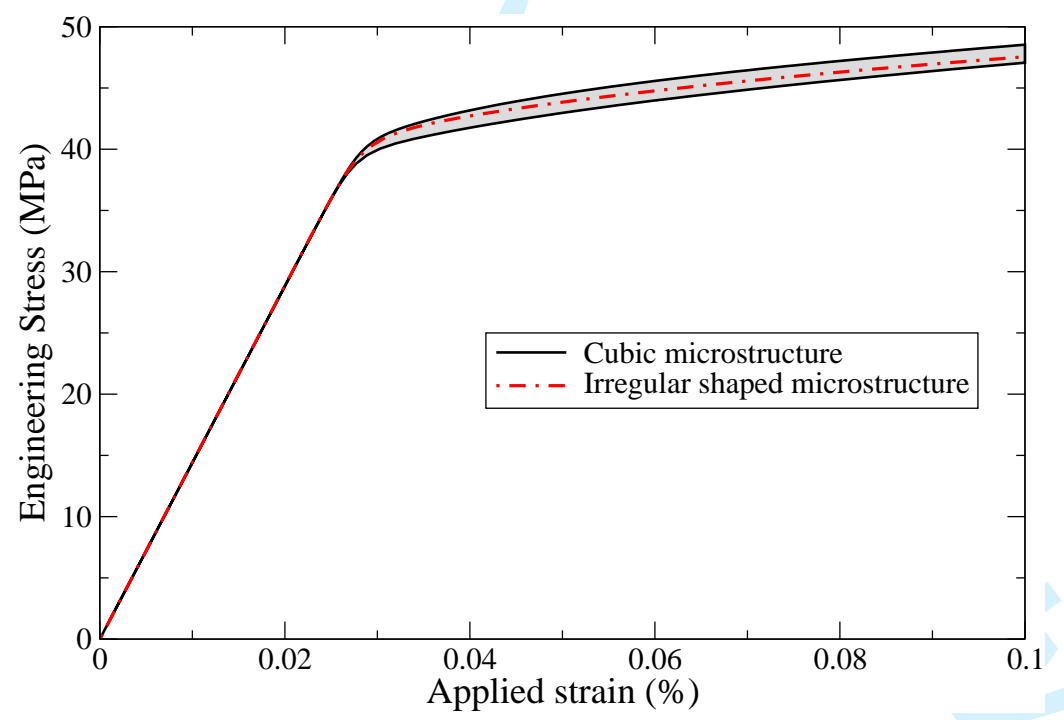

Fig. 4. The grey region represents the range of response curves for five sets of cube shaped microstructures as visualized in Figure 3d, showing the influence of different selections of grain textures. Lying within the scatter of these results the response is plotted for a model with the non-regular microstructure shown in Figure 3f.

The results from 5 tensile samples (with differently oriented grains) are represented through their stress-strain curves for both the regular (cubic grains, Figure 3d) and 
the non-regularized (Figure 3f) microstructural models, see Figure 4. Crystallographic slip is constrained at both internal grain boundaries and external specimen boundaries. Since the response of the non-regular configuration falls within the scatter of the responses of the regular cubic crystal models, the result appears to be hardly dependent of the crystal shape, and for the remainder of this paper, cubic shaped crystals will therefore be used. Beyond the initial linear part, the stressstrain curves during yielding diverge throughout a small range of stresses related to the statistical selection of grain orientations.

Inspired by the experimental results found in [22] and related comments in [28], two competing mechanisms that influence the size dependent behaviour are examined in detail, whereby the size of the grains in the microstructure is kept constant. As the dimensions of the structure diminish (accomplished by reducing the widths of the specimen), macroscopic deformation gradients are accentuated, while reversely, the deformation constraint imposed by the grain boundaries at adjacent grains decreases with decreasing dimensions. The influence of the grain boundaries are examined for the above defined cubic microstructures subjected to an applied tensile strain of $0.1 \%$. This applied macroscopic strain exceeds the physically intrinsic yield strain by a factor four, at which point, grains which are favourably oriented for slip will have endured at least some crystallographic slip. The stresses at an applied strain of $0.1 \%$ are compared in Figure 5 for the considered range of model widths (Figure 3) and the two previously outlined crystallographic slip boundary conditions. Furthermore, the standard crystal plasticity result is shown as well (no GNDs). Error bars representing 99\% confidence intervals were obtained from the different model texture variants and represent the expected scatter (mind the scaling on the vertical axis) due to different crystallographic grain orientations.

In spite of the relative large scales investigated, the influence of the crystallographic slip boundary conditions along the internal and external grain boundaries remains visible in the responses, presented by three almost parallel curves. The upper and lower curve represent the two extremes of the crystallographic slip boundary conditions, i.e. completely obstructed crystallographic slip across the grain boundary (leading to the highest stress response) and standard first-order crystal plasticity (which eases crystallographic slip at the grain boundaries, inducing the weakest response), respectively. An intermediate response is generated by assuming that the outer boundaries are free surfaces while restricting crystallographic slip at all internal grain boundaries, leading to a corresponding net decrease in the total number of GNDs compared to the fully restricted case. Localized strain gradients between adjacent grains develop, which increase the local GND densities, thereby contributing to an increase slip resistance. The overall back stress contribution is less pronounced.

The influence of the specimen width, while maintaining a constant microstructure with grain dimensions of $100 \times 100 \times 100 \mu \mathrm{m}$, is also evident from Figure 5 . For specimens exceeding $300 \mu \mathrm{m}$ in width, the two upper curves are expected to 
approach a macroscopic, near size independent response, while widths less than $300 \mu \mathrm{m}$ predict a reduction in flow strengths (at $0.1 \%$ strain). The origin of this size dependence is the decreasing interaction between neighbouring grains as the number of grains across the width decreases. With only a single grain across the width, there is no crystallographic constraint (in width direction). With an increasing number of grains, the number of constraints continues to increase until the macroscopic (plate-like) behaviour is reached. This trends holds for all cases, even though the first-order prediction will naturally predict a smaller stress (compared to the second-order predictions) because of the weaker grain boundary constraints. The error bars associated with each point correspond to the $99 \%$ confidence intervals associated with the multiple simulations for each specimen width. While the sample sizes were insufficiently large to generate statistical significant observations, they provide an indication of the relative influence of the grain sampling statistics. The error bar magnitudes over the range of widths investigated suggests that the limited number of grains across the specimen bears little influence on the scatter of the results. However, the number of grains in the length of the specimen does not change, and it seems that this is the dominant factor contributing to the error bars in Figure 5. Note that, the orientation of individual grains also contributes

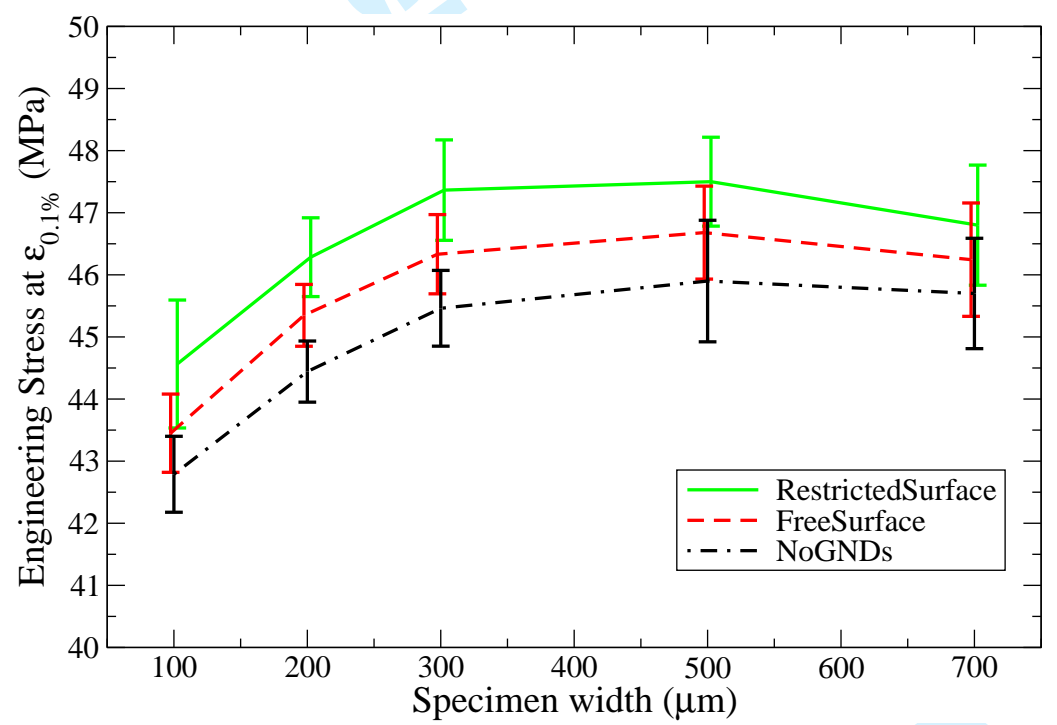

Fig. 5. Macroscopic tensile stresses at $0.1 \%$ strain comparing the influence of the crystallographic boundary conditions. The error bars represent $99 \%$ confidence intervals obtained for different local crystallographic orientations.

to the weakening trend observed in Figure 5, which simply results from the fact that the behaviour in a single cross-section is governed by a single grain rather than by an aggregate. The first-order effect and the second-order effect jointly contributed to the weakening effect predicted, where less grains and less grain boundaries are the physical origins. 


\subsection{The bending case}

While back stresses were found to contribute little to the macroscopic tensile response, they substantially do contribute under pure bending where a significant strain gradient develops. In the following, the configuration according to Figure $3 \mathrm{c}$ is subjected to bending. Varying the specimen thickness between 75 to $300 \mu \mathrm{m}$ by scaling the mesh in the thickness direction, generates a range of macroscopic through-thickness strain gradients which definitely have an impact on the normalized applied bending moments as plotted in Figure 6. In this figure, the normalized bending moments (scaled by the surface parameter $W T^{2}$ with $W$ the width and $T$ the thickness of the specimen) at an applied strain of $0.1 \%$ (at the outer fibre) are plotted versus the thickness. Unlike the macroscopic tensile responses plotted in Figure 5, the three different crystallographic boundary conditions appear to diverge with diminishing specimen thickness. When the crystallographic no slip boundary conditions across the grain boundaries are relaxed (along the specimen outer surface only, or overall) by specifying no GND accumulation on these boundaries, the models predict a slight decreasing trend with decreasing model thickness similar to the response in the tensile case. However, when the crystallographic slip is suppressed across all grain boundaries, including a passivating surface layer, the models predict an increasing normalized bending moment with decreasing thickness. This increased normalized bending moment results from the increasing throughthickness strain gradients in case of a diminishing thickness dimension. For the bending case, the first-order effect (weakening due to the reduction of grains) works opposite to the second-order effect (strengthening due to strain gradients). Depending on the local microstructural configuration, the overall trend may either show weakening or strengthening upon reducing the specimen size.

\section{Discussion}

The simulations presented highlight the importance of utilizing a material model capable of including the influence of second-order gradients for the simulation of the mechanical behaviour of micromanufactured components. While the simulations are qualitative in nature as they lack experimental verification, they indicate a range of geometrical sizes where first- and second-order effects will compete, leading to the trends observed in the present analysis. Disregarding the second-order gradients effects is found to lead to underestimating predictions of the macroscopic forces required during plastic deformation. The simulations also indicate that size effects in the examined range of scales are not only dependent on the external dimensions, but also on the ratio of the grain size with respect to the specimen size and the presence of macroscopic strain gradients.

The strengthening effect due to strain gradients results primarily from the back 
stresses associated with the GNDs required to satisfy lattice continuity. During monotonic loading, these back stresses will continue to resist plastic deformation by effectively reducing the driving force for crystallographic slip, however, the opposite is true when the loading direction is reversed. In that case, the back stresses associated with the dislocations will augment the driving force, producing the classic Bauschinger effect [5]. Therefore, the mechanical analysis of thin micromanufactured components subjected to strong strain gradients will have to include both isotropic and kinematic hardening mechanisms to accurately predict the mechanical behaviour.

By maintaining a constant grain size during the miniaturization simulations, decrease of the external dimensions also reduces the number of constituent grains that make up that part. This reduction in the number of crystals introduces a weakening effect due to the loss of crystallographic constraints between adjacent grains. This loss of constraints is adequately captured by a first-order crystal plasticity models, where the statistical influence of the total number of grains within a component is naturally accounted for. However, this weakening trend would not be captured using a conventional continuum plasticity model since the possibility to include the heterogeneity associated with individual grains of a crystalline material fails. Similarly, second-order continuum models that don't account for the contribution of individual grains such as [29] would also fail to capture this weakening trend with diminishing dimensions. However, by incorporation of the influence of strain gradients within a crystal plasticity framework, the resulting effect of concurrent strengthening and weakening can be described. 


\section{Conclusions}

Simulations using a strain-gradient crystal plasticity formulation of MEMS-like structures have been performed to examine the competition between first order weakening and gradient strengthening. The analysis focused on:

- A three-dimensional description of micromanufactured samples with a limited number of grains in a cross-section.

- A strain gradient crystal plasticity approach with an enriched back stress description that accounts for the internal dislocation induced stresses.

- A particular range of length scales for which a competition between first-order and second-order effects may be expected.

- The different sources that contribute to size effects: statistical effects, internal constraints (grain boundaries), external constraints and the externally applied load (possibly inducing macroscopic strain gradients).

More specifically the simulations revealed:

- In the presence of large macroscopic strain gradients, strain gradient strengthening dominates, requiring a greater applied load to overcome the increased internal back stresses associated with the GNDs in accordance with other researchers $[5,6,9,10,11,15]$.

- When macroscopic strain gradients are avoided, such as during uniaxial tension, the loss of microstructural constraints between adjacent grains dominates, resulting in a weakening trend with decreasing dimensions (miniaturization).

- The outer surface passivity has an obvious influence on the macroscopic behaviour of miniaturized components, especially when they are subjected to loading cases which induce large strain gradients.

- As dimensions diminish, a better qualitative agreement with the mechanical behaviour of micromanufactured parts (with a limited number of grains) are only possible on the basis of an enhanced crystal plasticity description, which accounts for the crystallographic orientations and volumes of each composing grain and in which GND-driven strain gradient strengthening is included in the slip resistance and the back stress calculation.

\section{Acknowledgements}

This work was supported by the Netherlands Institute of Metals Research (NIMR) under project MC2_01096. 


\section{References}

[1] World Technology Evaluation Center. International assessment of research and development in micromanufacturing. Technical report, National Science Foundation, USA, October 2005.

[2] S. R. Kalidindi, C. A. Bronkhorst, and L. Anand. Crystallographic texture evolution in bulk deformation processing of fcc metals. J. Mech. Phys. Solids, 40:537-569, 1992.

[3] R. J. Asaro and A. Needleman. Overview no. 42 texture development and strain hardening in rate dependent polycrystals. Acta Metall., 33(6):923-953, 1985.

[4] M. F. Ashby. The deformation of plastically non-homogeneous alloys. Philos. Mag., 21:399-424, 1970.

[5] C. J. Bayley, W. A. M. Brekelmans, and M. G. D. Geers. A comparison of dislocation induced back stress formulations in strain gradient crystal plasticity. Int. J. Solids Struct., Accepted, 2006.

[6] L. P. Evers, W. A. M. Brekelmans, and M. G. D. Geers. Non-local crystal plasticity model with intrinsic SSD and GND effects. J. Mech. Phys. Solids, 52:2379-2401, 2004.

[7] C. S. Han, H. Gao, Y. Huang, and W. D. Nix. Mechanism-based strain gradient crystal plasticity - I. theory. J. Mech. Phys. Solids, 53:1188-1203, 2005.

[8] C. S. Han, H. Gao, Y. Huang, and W. D. Nix. Mechanism-based strain gradient crystal plasticity - II. analysis. J. Mech. Phys. Solids, 53:1204-1222, 2005.

[9] N. A. Fleck, G. M. Muller, M. F. Ashby, and J. W. Hutchinson. Strain gradient plasticity: theory and experiment. Acta Metall. Mater., 41:475-487, 1994.

[10] J. S. Stölken and A. G. Evans. A microbend test method for measuring the plasticity length-scale. Acta Mater., 46:5109-5115, 1998.

[11] L. P. Evers, W. A. M. Brekelmans, and M. G. D. Geers. Scale dependent crystal plasticity framework with dislocation density and grain boundary effects. Int. J. Solids Struct., 41:5209-5230, 2004.

[12] P. Gudmundson. A unified treatment of strain gradient plasticity. J. Mech. Phys. Solids, 52(6):1379-1406, 2004.

[13] P. Fredriksson and P. Gudmundson. Size-dependent yield strength of thin films. Int. J. Plast., 21:1834-1854, 2005.

[14] G. Z. Voyiadjis and R. Abu Al-Rub. Gradient plasticity theory with a variable length scale parameter. Int. J. Solids Struct., 42:3998-4029, 2005.

[15] M. E. Gurtin. A gradient theory of single-crystal viscoplasticity that accounts for geometrically necessary dislocations. J. Mech. Phys. Solids, 50:5-32, 2002.

[16] M. Zaiser and E. C. Aifantis. Geometrically necessary dislocations and strain gradient plasticity: a dislocation dynamics point of view. Scripta Mat., 48(2):133-139, 2003.

[17] L. Nicola, E. van der Giessen, and A. Needleman. Size effects in polycrystalline thin films analyzed by discrete dislocation plasticity. Thin Solid Films, 
479(1-2):329-338, 2005.

[18] S. Yefimov and E. van der Giessen. Multiple slip in a strain-gradient plasticity model motivated by a statistical-mechanics description of dislocations. Int. J. Solids Struct., 42(11-12):3375-3394, 2005.

[19] S. Miyazaki, K. Shibata, and H. Fujita. Effect of specimen thickness on mechanical properties of polycrystalline aggregates with various grain sizes. Acta Metall., 27:855-862, 1978.

[20] R.T.A. Kals and R. Eckstein. Miniaturization in sheet metal working. J. Mat. Proc. Tech., 103:95-101, 2000.

[21] M. Klein, A. Hadrboletz, B. Weiss, and G. Khatibi. The 'size effect' on the stress-strain, fatigue and fracture porperties of thin metallic foils. Mat. Sci. Eng. A, 319-321:924-928, 2001.

[22] P. J. M. Janssen, Th. H. de Keijser, and M. G. D. Geers. An experimental assessment of grain size effects in the uniaxial straining of thin Al sheet with a few grains across the thickness. Mat. Sci. Eng. A, 419:238-248, 2006.

[23] P. Franciosi and A. Zaoui. Multislip in F.C.C. crystals a theoretical approach compared with experimental data. Acta Metall., 30:1627-1637, 1982.

[24] A. Arsenlis and D. M. Parks. Modeling the evolution of crystallographic dislocation density in crystal plasticity. J. Mech. Phys. Solids, 50:1979-2009, 2002.

[25] A. H. Cottrell. Dislocation and plastic flow in crystals. Oxford University Press, 1952.

[26] J. Harder. A crystallographic model for the study of local deformation processes in polycrystals. Int. J. Plast., 15:605-624, 1999.

[27] D. Stojkovic. Strain gradient crystal plasticity: on the influence of configurational variations and size in tension and bending. Internal report, Eindhoven University of Technology, 2004.

[28] M. G. D Geers, W. A. M. Brekelmans, and P.J.M. Janssen. Size effects in miniaturized polycrystalline fcc samples: strengthening versus weakening. Int. J. Solids Struct., Accepted, 2006.

[29] Y. Huang, H. Gao, W. D. Nix, and J. W. Hutchinson. Mechanism-based strain gradient plasticity-II. Analysis. J. Mech. Phys. Solids, 48:99-128, 2000. 


\section{Pagelillofophical Magazine \& Philosophical Magazine Letters}


Philosophical Magazine \& Philosophical Magragiaepatters

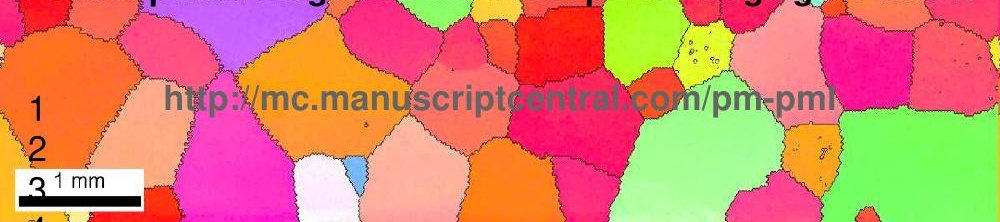




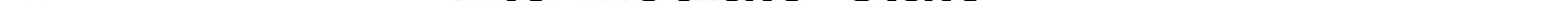




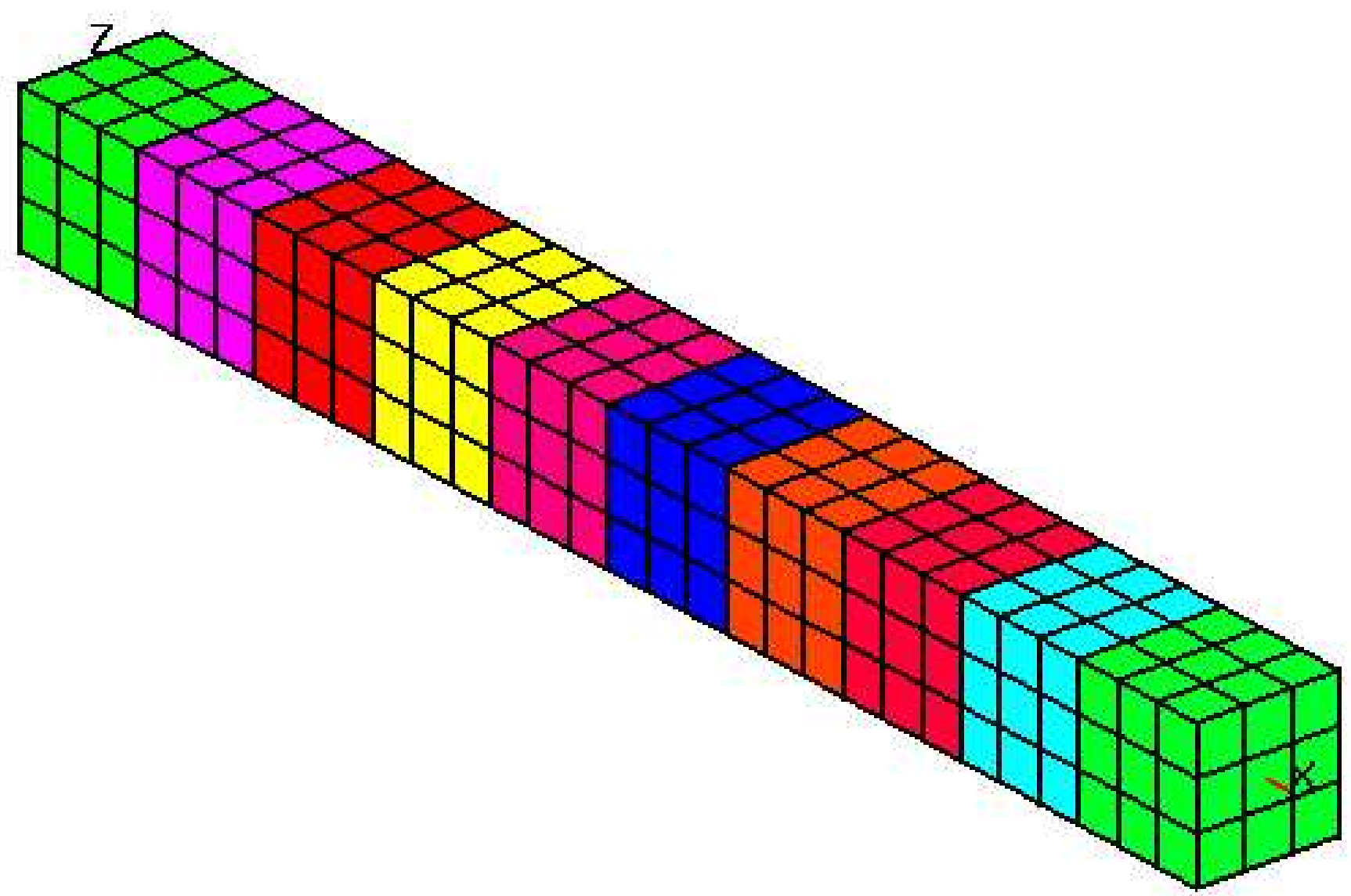




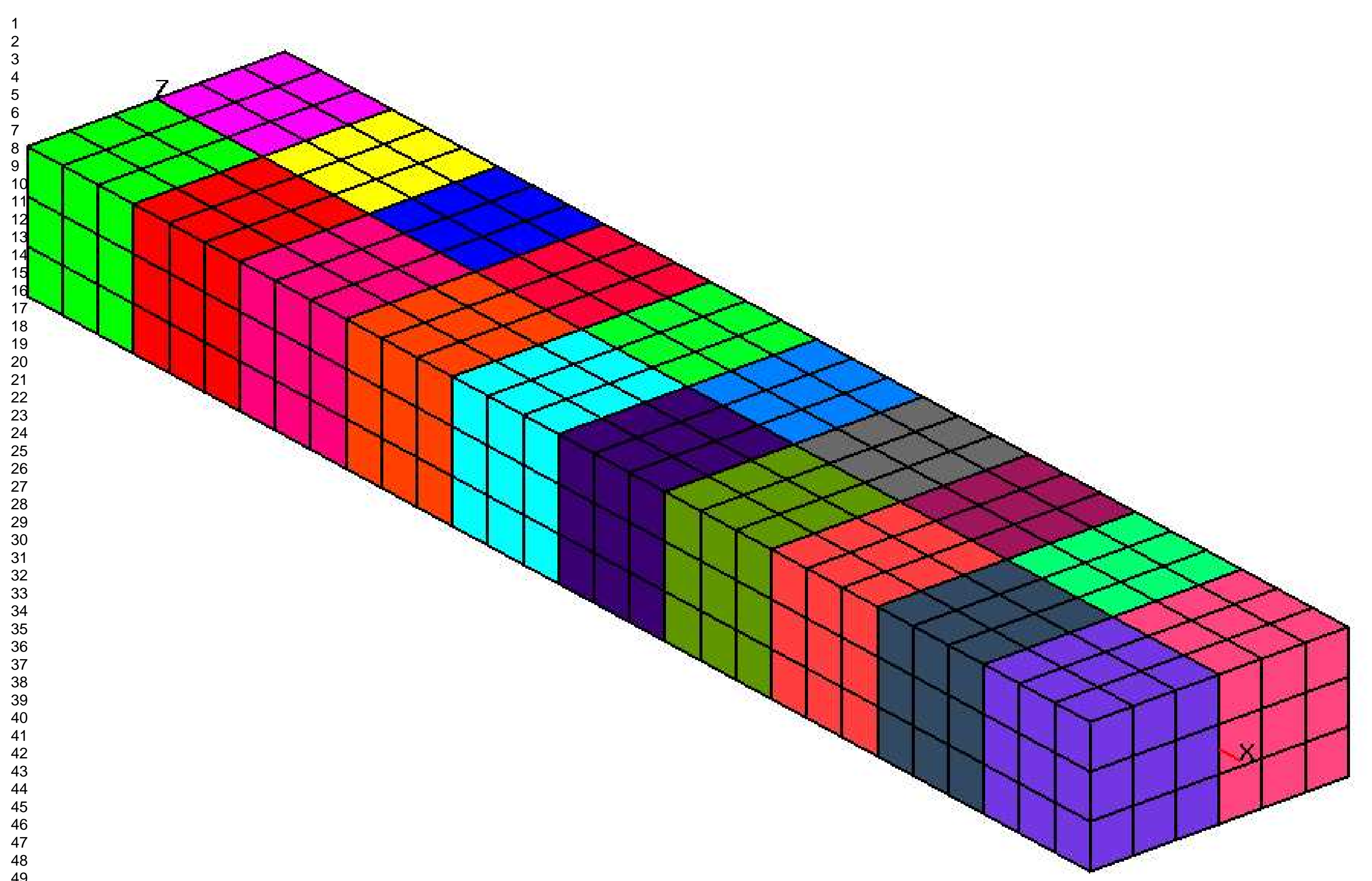




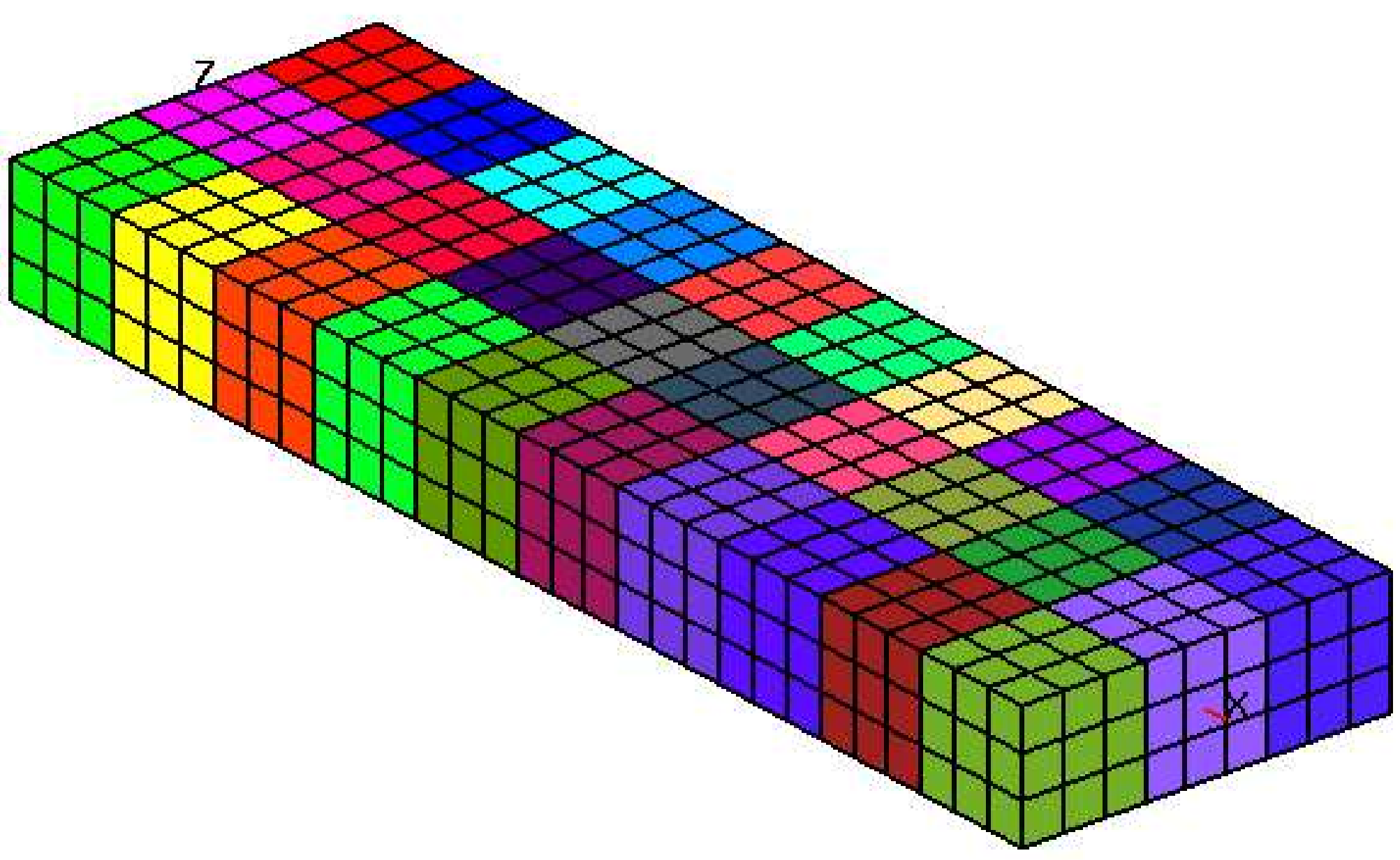




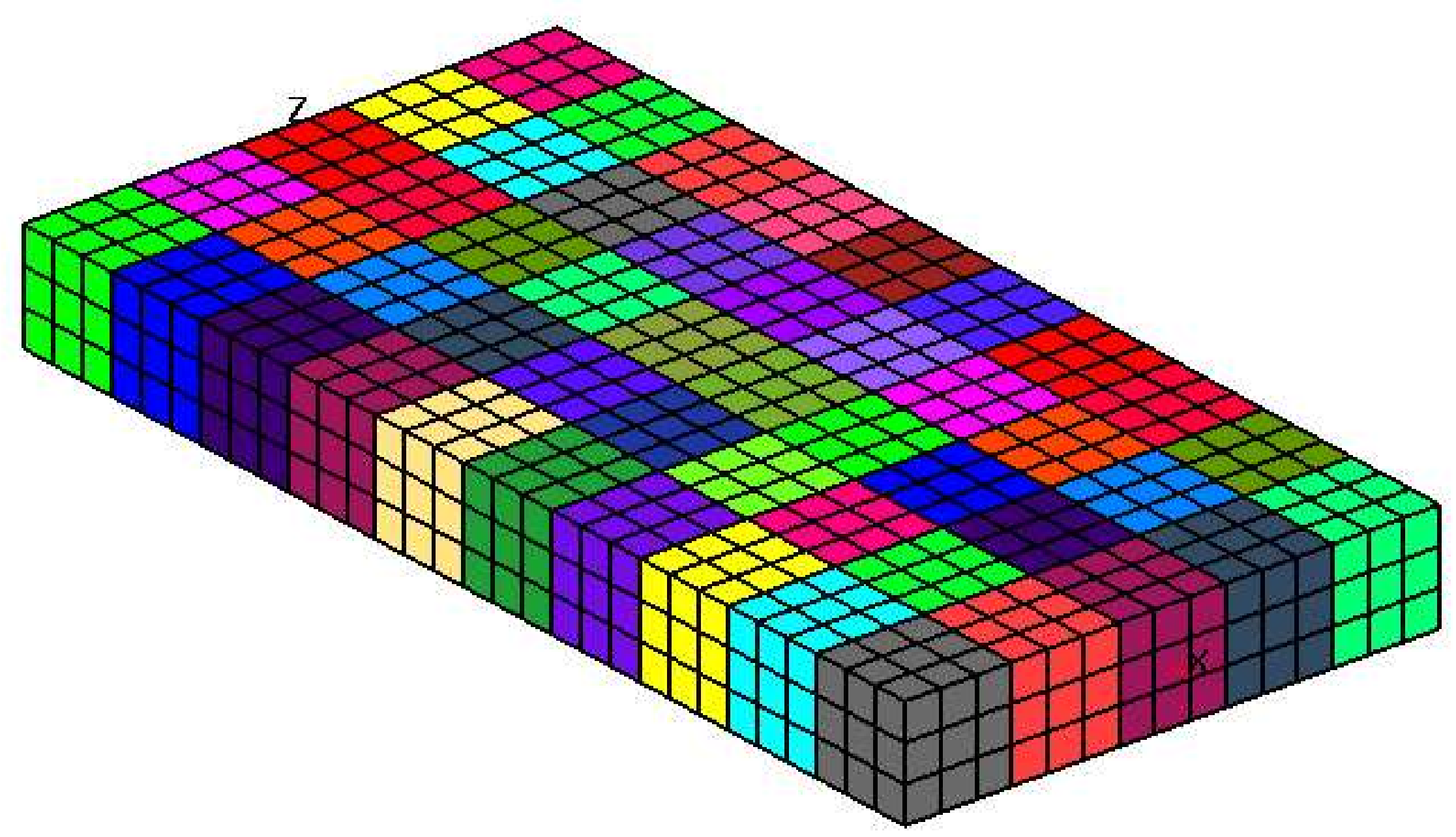




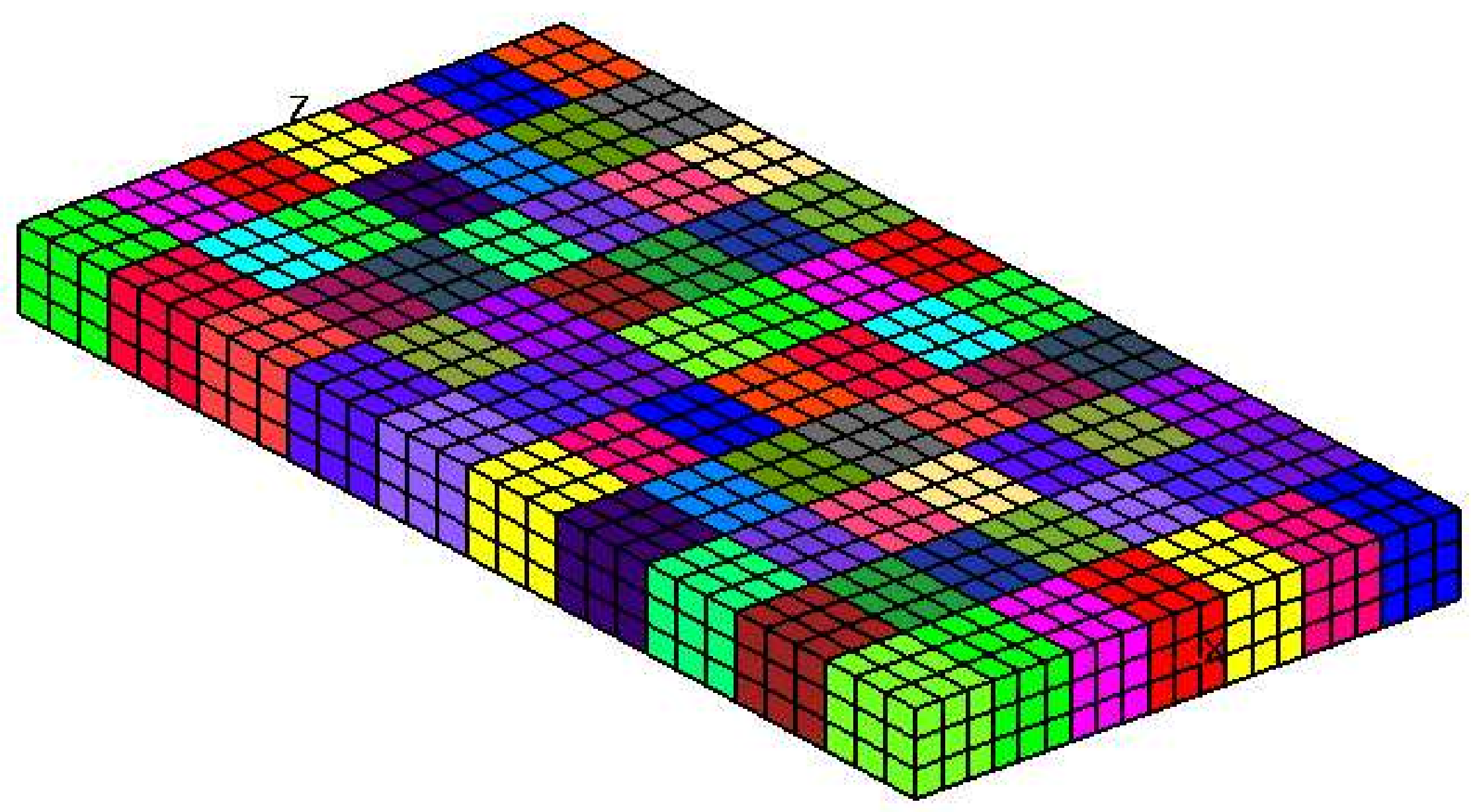




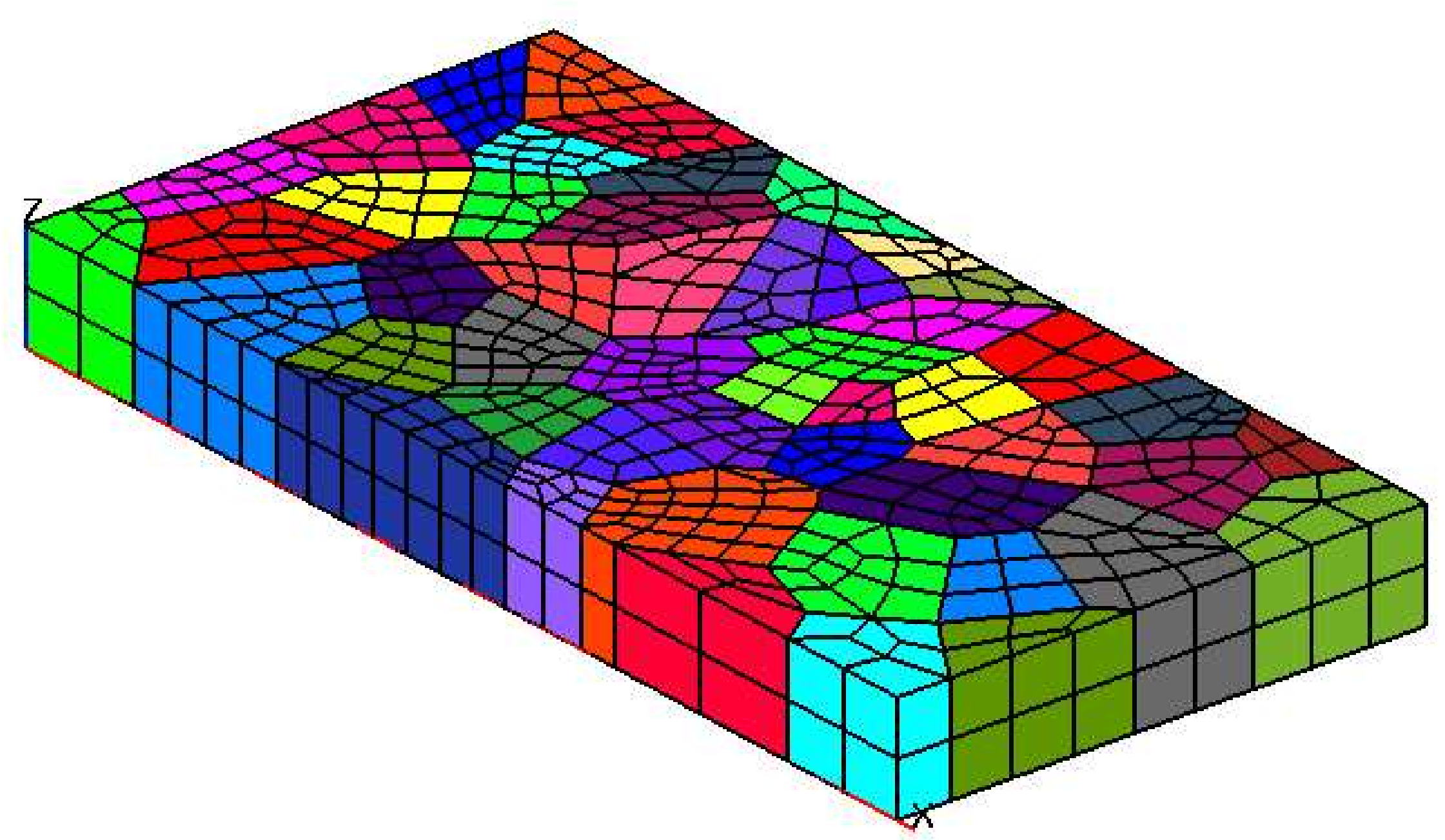

http://mc.manuscriptcentral.com/pm-pml 


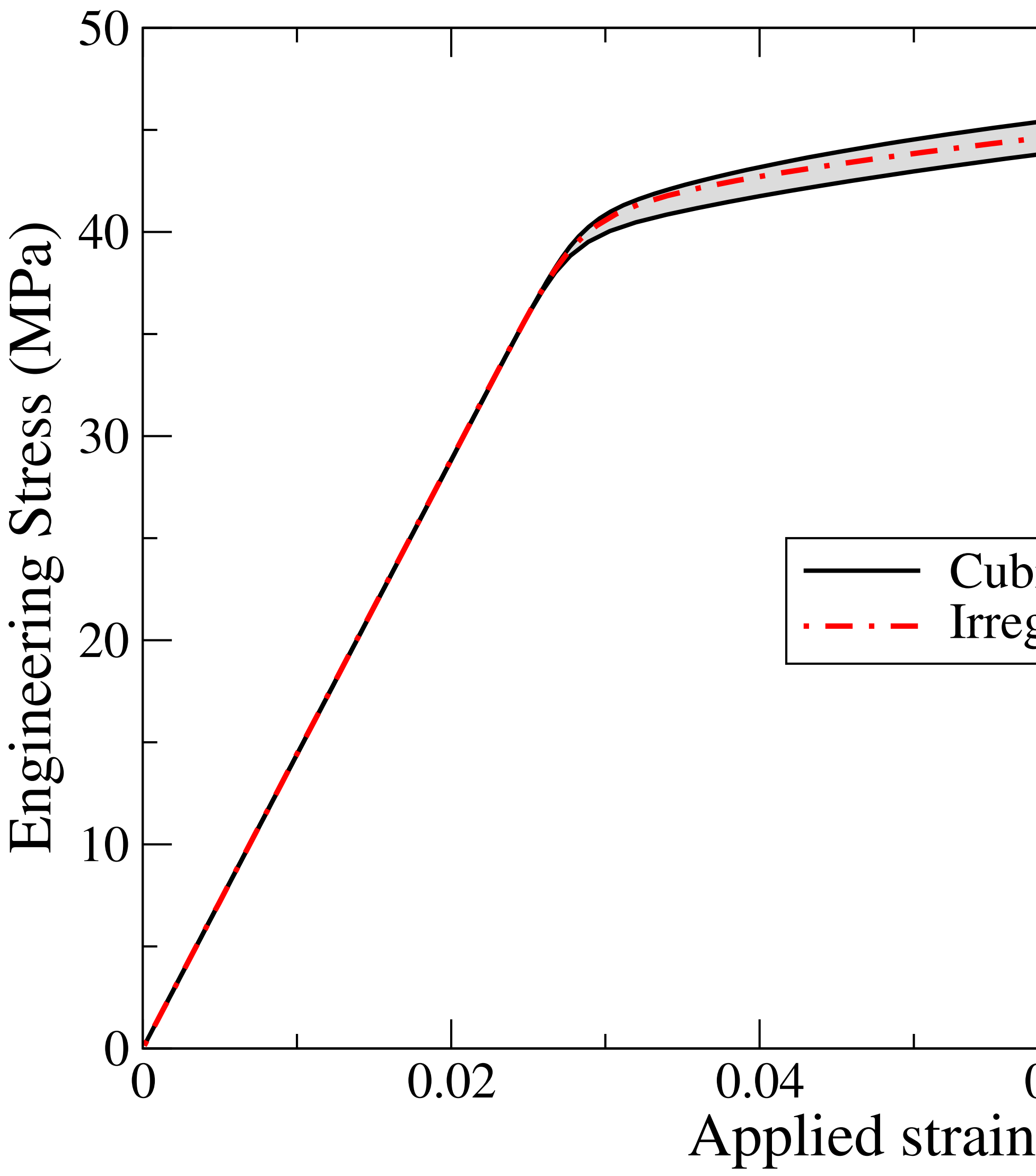




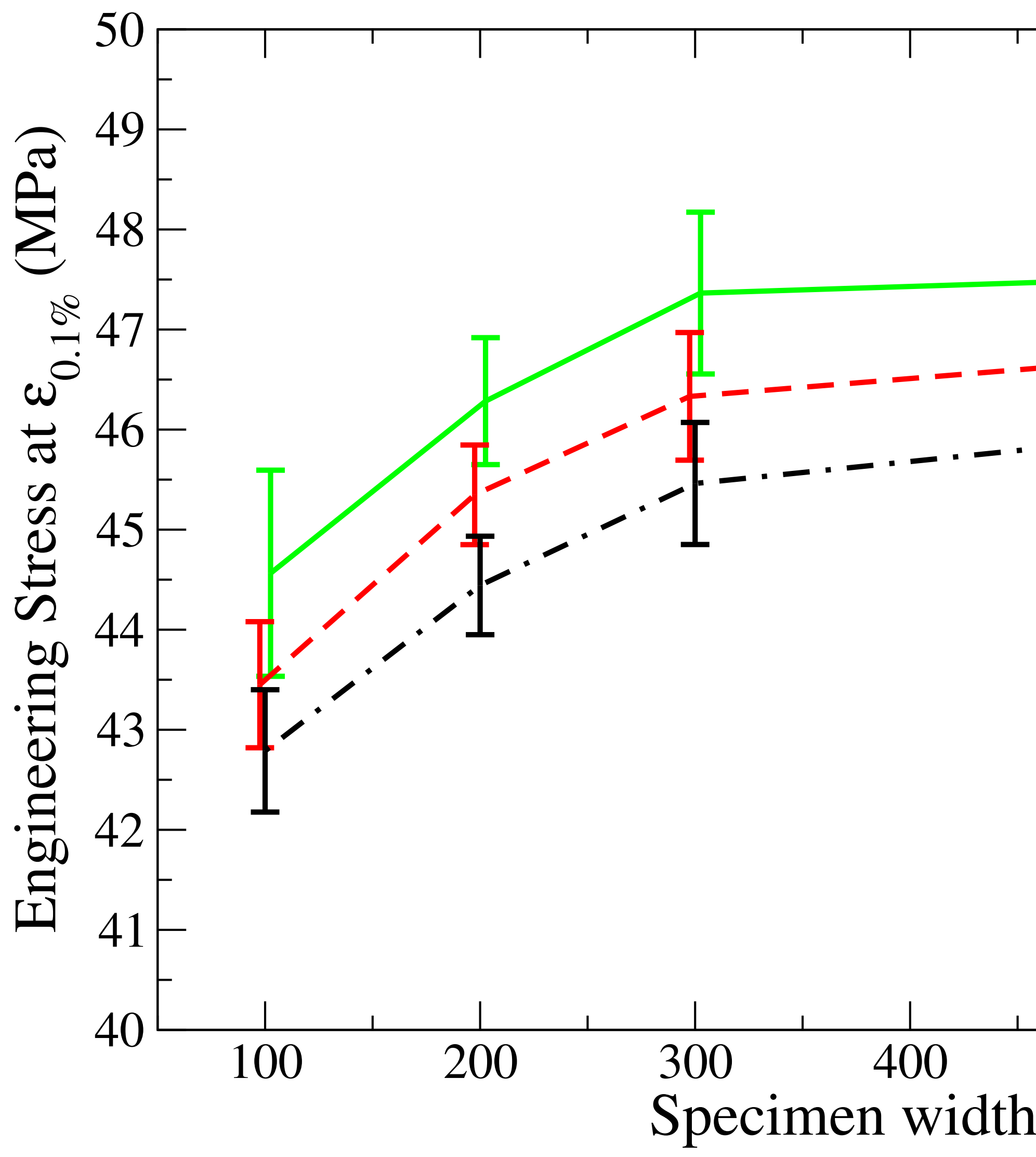

http://mc.manuscriptcentral.com/pm-pml 


3

3

32

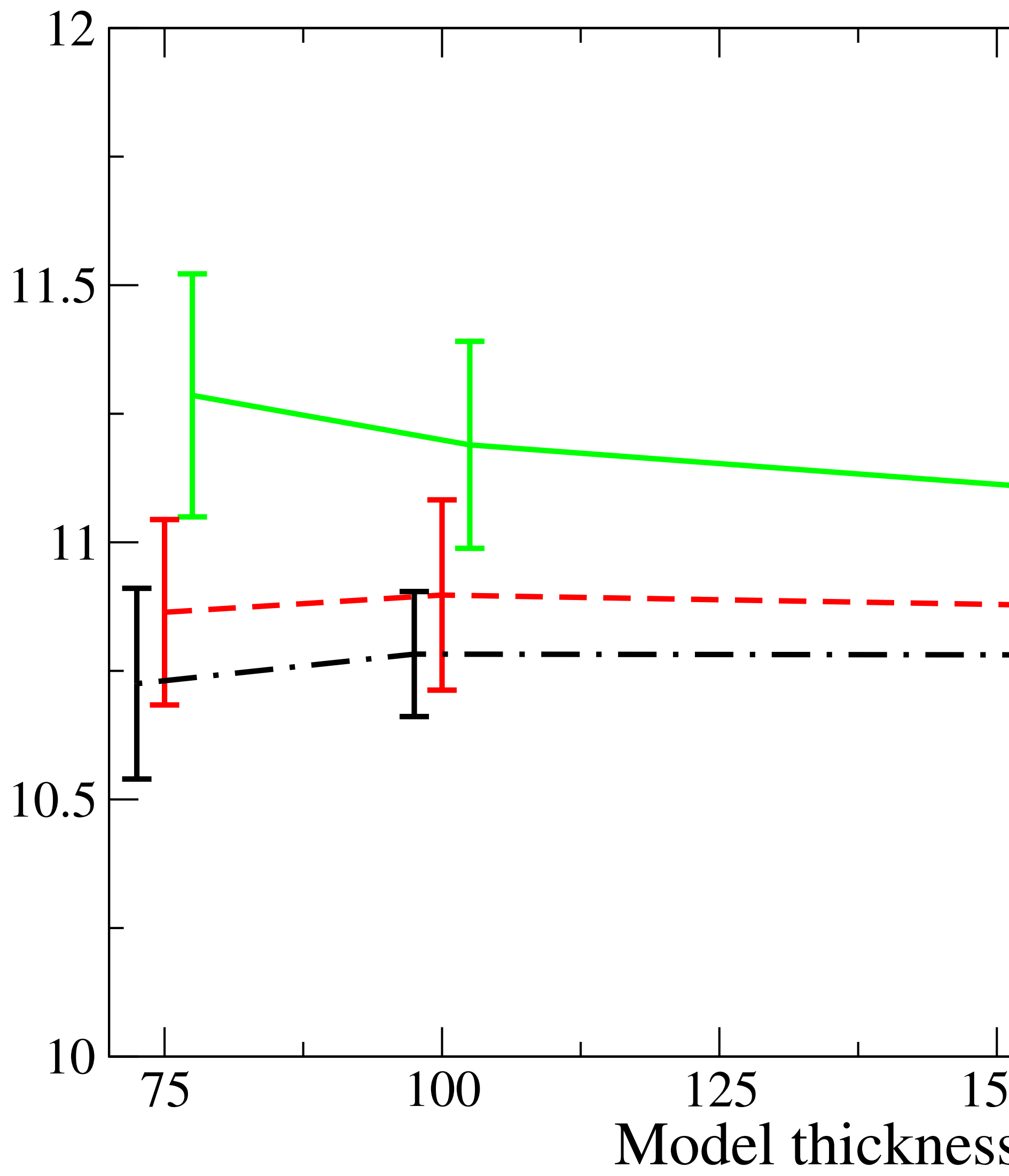

